\title{
Inhibitory control mediates a negative relationship between body mass index and intelligence: A neurocognitive investigation
}

\author{
L. Faul $^{1}$ - N. D. Fogleman ${ }^{1} \cdot$ K. M. Mattingly ${ }^{1}$ - B. E. Depue ${ }^{1,2}$ \\ Published online: 6 February 2019 \\ (C) The Psychonomic Society, Inc. 2019
}

\begin{abstract}
The structure and function of the human brain is closely related to cognitive processes of the mind and physiological processes of the body, suggesting that an intricate relationship exists between cognitive health, body health, and underlying neural architecture. In the current study, morphometric differences in cortical and subcortical gray matter regions, white matter integrity, and resting-state functional connectivity was assessed to determine what combinations of neural variables best explain an interconnected behavioral relationship between body mass index (BMI), general intelligence, and specific measures of executive function. Data for 82 subjects were obtained from the Nathan Kline Institute Rockland Sample. Behavioral results indicated a negative relationship between BMI and intelligence, which exhibited mediation by an inhibitory measure of executive function. Neural analyses further revealed generally contrasting associations of BMI, intelligence, and executive function with cortical morphometric regions important for inhibitory control and directed attention. Moreover, BMI related to morphometric alterations in components of a frontolimbic network, namely reduced thickness in the anterior cingulate cortex and ventromedial prefrontal cortex, whereas intelligence and inhibitory control primarily related to increased thickness and volume in parietal regions, as well as significantly increased across-network connectivity of visual and default mode resting-state networks. These results propose that medial prefrontal structure and interconnected frontolimbic and frontoparietal networks are important to consider in the relationship between BMI, intelligence, and executive function.
\end{abstract}

Keywords Body mass index $\cdot$ Intelligence $\cdot$ Executive function $\cdot$ Inhibitory control $\cdot$ Morphometry $\cdot$ Resting-state fMRI

\section{Introduction}

The brain is considered to be the most complex organ in the human body, which stems from intricate networks of structural and functional neural features that must maintain a diverse cognitive system. The field of cognitive neuroscience has consistently studied the relationship between the brain and behavior in an effort to better understand the importance and utilization of different brain regions and, more importantly, widespread neural networks. During the past two decades,

Electronic supplementary material The online version of this article (https://doi.org/10.3758/s13415-019-00695-2) contains supplementary material, which is available to authorized users.

B. E. Depue

brendan.depue@louisville.edu

1 Department of Psychological and Brain Sciences, University of Louisville, Louisville, KY, USA

2 Department of Anatomical Sciences and Neurobiology, University of Louisville, Louisville, KY, USA researchers also have come to increasingly consider the inter-reliance and dependency of the brain and body, based on numerous studies documenting the association of physical health (e.g., exercise) with cognitive function (Cotman \& Berchtold, 2002; Cotman, Berchtold, \& Christie, 2007; Hamer \& Chida, 2009; Hillman, Erickson, \& Kramer, 2008), as well as psychological factors that contribute to a healthy or unhealthy body state (Cohen \& Herbert, 1996; Rozanski, Blumenthal, \& Kaplan, 1999; Trost, Owen, Bauman, Sallis, \& Brown, 2002). Although the human brain underlies the complex cognitive processes of the mind, the structure and function of the brain also is closely tied to physiological processes of the body. However, few studies have fully examined the intricate connections between body, brain, and behavior.

Investigating physiological health disorders and their corresponding associations with neural features, such as cortical morphometry or functional connectivity, can help to clarify these relationships by elucidating distinct changes in neural systems that accompany differences in physiology and cognition. Research examining obesity, known to accompany a 
wide range of health complications, including high blood pressure, high cholesterol levels, arthritis, diabetes, and cancer, is a primary example (Kopelman, 2000, 2007; Mokdad et al., 2003). Although obesity can be classified based on a variety of measurements, the World Health Organization (WHO, 2000) uses body mass index (BMI) as a "crude population measure of obesity." Body mass index often is calculated as kilograms per square meter and therefore incorporates both the height and weight of an individual (Keys, Fidanza, Karvonen, Kimura, \& Taylor, 1972).

Studies have shown that BMI measurements in the obese range associate with smaller whole brain and total grey matter volume compared with individuals in the normal or overweight BMI range (Gunstad et al., 2008; Yokum, Ng, \& Stice, 2012). Obese individuals also tend to show reduced cortical thickness and volume in frontal (i.e., dorsal anterior cingulate cortexdACC - and medial orbitofrontal cortex) and parietal (i.e., posterior parietal cortex) regions (Hassenstab et al., 2012; Marqués-Iturria et al., 2013; Raji et al., 2010). Furthermore, obesity is associated with reduced fractional anisotropy (FA) - a measure of white matter structural integritythroughout the brain in areas, such as the corpus callosum and fornix (Karlsson et al., 2013; Stanek et al., 2011). High BMI relates to differences in executive function (EF) and reward networks when assessed with functional magnetic resonance imaging (fMRI) during resting-state (Kullmann et al., 2012; Lips et al., 2014). These observed morphometric and functional differences in obesity align with findings of elevated BMI and decreased blood flow in the prefrontal cortex (Willeumier, Taylor, \& Amen, 2011), suggesting some changes to the brain may result from physiological factors affected by obesity.

It is worth emphasizing that BMI is an indirect measure of body physiology and is limited in its ability to truly classify an individual as overweight or obese. Individual variations in neural features across a broad range of BMI measures therefore is worth considering to determine correlational relationships with the brain, as opposed to grouping individuals in either high or low BMI. Increased BMI significantly relates to decreased global brain volume (Ward, Carlsson, Trivedi, Sager, \& Johnson, 2005), as well as decreased thickness and volume in frontal, parietal, and occipital regions (Boyle et al., 2015; Kennedy, Collins, \& Luciana, 2016; Taki et al., 2008; Veit et al., 2014; Walther, Birdsill, Glisky, \& Ryan, 2010), including the right ventromedial prefrontal cortex: vmPFC (Medic et al., 2016). Similarly, increases in BMI over a 1year period correlate with decreases in cortical volume of the superior and middle frontal gyri among female adolescents (Yokum et al., 2012). Increased BMI also associates with a global reduction of white matter structural integrity (Verstynen et al., 2012) and reduced integrity specifically in the corpus callosum and fornix $(\mathrm{Xu}, \mathrm{Li}, \mathrm{Lin}, \mathrm{Sinh} a$, \& Potenza, 2013). Additionally, higher BMI relates to functional connectivity deficits within sensory integration and attention networks important for sensorimotor and visual processing (Doucet, Rasgon, McEwen, Micali, \& Frangou, 2017) and lowered metabolic activity in the prefrontal cortex (Volkow et al., 2009). Thus, even when looking at correlational relationships that include individuals in a healthy BMI range, similar results are observed to those comparing obese and nonobese subjects, especially for cortical morphometry measures in frontal regions.

These widespread associations of BMI with the brain suggest that it is important to consider cognitive functions that may relate to body physiology. One way to begin such an investigation is to first understand how common cognitive measures of the mind independently relate to brain structure and function. Intelligence is arguably one of the most wellknown measures of cognitive functioning given its long history of association with education, social status, and a wide variety of other performance measures (Gottfredson, 1998; Neisser et al., 1996). Spearman first introduced the concept of general intelligence $(g)$ to explain how individuals consistently display high levels of cognitive ability on a range of mental assessments (Spearman, 1904), which preceded the intelligence quotient (IQ), a number that quantifies performance on these tests and functions to measure one's intellectual ability. Just as BMI serves as a general indicator of the body, intelligence also serves as a general indicator of the mind's ability to perform cognitive processes. Moreover, fluid intelligence scores that reflect adaptive thinking can increase within an individual following training with working-memory tasks (Au et al., 2015; Jaeggi, Buschkuehl, Jonides, \& Perrig, 2008), emphasizing its representation of dynamic cognitive ability that can be enhanced over time.

Regarding neural relationships, increased intelligence generally has a positive association with whole brain grey and white matter volume (Haier, Jung, Yeo, Head, \& Alkire, 2004; McDaniel, 2005; Wickett, Vernon, \& Lee, 2000; Witelson, Beresh, \& Kigar, 2006). Increased intelligence also has been associated with broad increases in cortical thickness across frontal and parietal regions (Menary et al., 2013), as well as increased functional connectivity in neural networks incorporating these same regions (Song et al., 2008). Combining structural and functional studies, researchers have developed the Parieto-Frontal Integration Theory (P-FIT) of Intelligence, which proposes that general intelligence requires efficient communication among networks consisting of the dorsolateral prefrontal cortex (dIPFC) and anterior cingulate cortex (ACC) in association with sensorimotor regions extending into the parietal lobe (Colom et al., 2009; Jung \& Haier, 2007; Vakhtin, Ryman, Flores, \& Jung, 2014). P-FIT suggests an underlying structural and functional neural system that is strengthened with increasing intelligence. Interestingly, this generally positive relationship between intelligence and brain measures appears incongruent with that of increased 
BMI, especially in regard to cortical morphometric features. Furthermore, these contrasting associations with the brain complement several behavioral studies demonstrating lower general intelligence related to higher BMI, especially when considering childhood intelligence as a predictor for adult obesity (for a review, see Kanazawa, 2014). However, a direct assessment of how other cognitive and neural factors might influence such a relationship remains lacking.

In addition to intelligence, more specific measures of cognitive function also relate to physiological health. For example, multiple EF constructs that control and measure regulatory behavior are negatively associated with increased BMI (Gunstad et al., 2007; Walther et al., 2010), suggesting that differences in general intelligence measures might merely reflect these particular relationships. Whereas intelligence scores provide a global assessment of cognitive function, measures of EF can more specifically assess goal maintenance (working memory), attentional focus, and inhibitory control (Friedman et al., 2006; Harrison et al., 2013; Heitz et al., 2006). Accordingly, the evolution of the human brain is closely tied to increases in intelligence as an indicator of progressively more complex and integrated EFs (Roth \& Dicke, 2005). When taken together, EFs and general intelligence ability both reflect a top-down control system mapping to frontoparietal cortical connections and, more specifically, global connectivity of the PFC (Cole, Yarkoni, Repovs, Anticevic, \& Braver, 2012). Hence, the regions responsible for integrating information promote intelligent goal-directed behavior (Barbey et al., 2012) and flexible shifts in cognitive control (Cole et al., 2013). In contrast, increased BMI generally associates with metabolic, structural, and functional deficits in these same regions, possibly relating to deficits in topdown cognitive function. Specifically, reduced inhibitory control ability in individuals with high BMI has been shown to mediate decreased cortical thickness in the PFC (Lavagnino et al., 2016), suggesting that high BMI relates to a weakened executive and P-FIT system.

The neural correlates involving BMI, intelligence, and EF therefore suggest that common structural and functional features within the brain might help to explain a compelling relationship between the brain, body physiology, and cognitive function. Importantly, such an assessment not only adds to a growing literature on structural, functional, and behavioral associations but also clarifies specific relationships between physical and mental health. Thus, we examined the neural correlates of a general indicator of body health (i.e., BMI) and a general indicator of cognition (i.e., intelligence) to investigate the mind-body connection between these two measures. We further assessed the possibility of EFs as potential mediators for this relationship, given that an EF assessment can suggest more specific cognitive abilities that relate to these general measures. Our analyses assessed surface-based cortical and subcortical morphometry, as well as indicators of white matter tract integrity (fractional anisotropy [FA]) to identify morphometric features that relate to BMI, intelligence, and EF measures. In addition, behaviorally relevant intrinsic connectivity networks (ICNs) were assessed using resting-state fMRI analysis. Based on findings from previous literature, we hypothesized that common structural and functional neural networks will help to explain the relationship between BMI, intelligence, and EF. More specifically, we hypothesized that a negative relationship between BMI and intelligence is mediated by EF ability, which primarily manifests as differing morphometric associations with frontal and parietal cortical regions. We argue that utilizing a comprehensive structural and functional analysis may elucidate additional interactions between BMI, intelligence, and EF that otherwise would be overlooked if we only investigated one type of neural feature.

\section{Methods}

The data used in this investigation were obtained from the 1000 Functional Connectomes Project (FCP) database (http://fcon_1000.projects.nitrc.org). The FCP was created in 2009 and joined with the International Neuroimaging DataSharing Initiative (INDI) to provide public access to large datasets, with a focus on resting-state fMRI data (Mennes, Biswal, Castellanos, \& Milham, 2013). In particular, the original Nathan Kline Institute Rockland Sample (NKI-RS) is a prospectively shared dataset that offers data from individuals aged 4 to 85 years and includes a wide range of phenotypic information for all subjects, as well as anatomical, diffusionweighted imaging (DWI), and resting-state fMRI scans (Nooner et al., 2012). Lavagnino et al. (2016) used the NKIRS dataset in a recent publication on the association between BMI and inhibitory control.

\section{Participants}

Demographic characteristics are provided in Table 1. A total of 82 adults (53 males, 29 females), $18-55$ years of age ( $M=$ $33.2, S D=10.9$ ), were included in this study. Subjects from the NKI-RS younger than age 18 or older than age 55 years were excluded from the analyses to account for confounding factors related to development or aging of the brain. Inclusion criteria required the availability of behavioral and neuroimaging data (if most of the NKI-RS battery was completed), as well as proper segmentation of structural data for cortical and subcortical analyses.

\section{Behavioral assessments}

Of primary interest for this study were BMI measurements and scores on the Wechsler Abbreviated Scale of 
Table 1 Demographics

\begin{tabular}{llll}
\hline Characteristic/Measure & M & SD & Range \\
\hline Age & 33.2 & 10.9 & $18-55$ \\
BMI & 26.7 & 5.3 & $17.3-40.5$ \\
WASI Full Scale & 110 & 12.7 & $84-138$ \\
WASI Vocabulary & 54.9 & 9.2 & $33-75$ \\
WASI Similarities & 55.3 & 8.1 & $39-72$ \\
D-KEFS CWIT* & 10.9 & 2.6 & $3-17$ \\
\hline
\end{tabular}

$\mathrm{N}($ Females $)=29 ; \mathrm{N}($ Males $)=53 . *$ The Color-Word Interference Test (CWIT) was the only D-KEFS measure from the NKI-RS that significantly related to both intelligence and BMI (see Behavioral Analyses)

Intelligence, Second Edition (WASI; Wechsler, 2011), and Delis-Kaplan Executive Function System (D-KEFS; Delis, Kaplan, \& Kramer, 2001). All participants had BMI measurements $(M=26.7, S D=5.3)$, as well as scaled scores on the WASI (Full $M=110, S D=12.7$ ) and various D-KEFS subtests. BMI is calculated by dividing the weight of an individual by the square of their height and is reported in $\mathrm{kg} / \mathrm{m}^{2}$. The NKI-RS used the WHO definition for BMI (WHO, 2000), such that participants with a BMI less than 18.50 were characterized as underweight, 18.50-24.99 as normal, 25.0029.99 as overweight, and anything greater than 30.00 as obese. However, we did not group subjects based on this classification, as we assessed correlational relationships across the full range of BMI measures.

The WASI is an abbreviated measure of cognitive intelligence and is comprised of four main subtests administered in the following order: block design, vocabulary, matrix reasoning, and similarities (Wechsler, 2011). Block design and matrix reasoning subtests form the perceptual reasoning index (PRI), and vocabulary and similarities subtests form the verbal comprehension index (VCI). During the block design subtest, subjects must recreate two-dimensional geometric designs using three-dimensional cubes, thereby testing fluid intelligence and visual perception. Similarly, the matrix reasoning subtest evaluates fluid and visual intelligence by requiring examinees to complete a matrix, or puzzle, by selecting the appropriate choice among a set of options. The vocabulary subtest measures verbal and crystallized intelligence and does so by instructing the examinee to define a word or concept that is presented to them. Likewise, the similarities subtest evaluates abstract verbal reasoning abilities by requiring subjects to describe the relationship between two objects or concepts. The NKI-RS data included a WASI full scale score, as well as scores on each individual WASI subtest. The full D-KEFS comprises nine standardized tests that measure specific components of EF; these include the Trail Making, Verbal Fluency, Design Fluency, Color-Word Interference, Sorting, Twenty Questions, Word Context, Tower, and Proverb tests (Delis et al., 2001). However, only scores on the Verbal
Fluency, Design Fluency, Color-Word Interference, Sorting, and Twenty Questions were available for this analysis.

\section{Behavioral analyses}

All analyses controlled for age, sex, and socioeconomic status (SES), based on previous research demonstrating a significant contribution of these factors to individual differences in BMI and cognitive functioning (Ball \& Crawford, 2005; Haier, Jung, Yeo, Head, \& Alkire, 2005; Sirin, 2005; Ursache \& Noble, 2016; Wang \& Beydoun, 2007; Yi, Ohrr, Shin, \& Yi, 2015). SES was measured with the Hollingshead Four-Factor Index of Socioeconomic Status, which takes into account marital status, retired/employed status, educational attainment, and occupational prestige (Hollingshead, 1975).

Pearson correlations were conducted to assess patterns of relations among measures. Feature selection was based on identifying significant correlational relationships between BMI, WASI, and all available D-KEFS measures that were provided by the NKI-RS (Verbal Fluency, Design Fluency, Color-Word Interference, Sorting, and Twenty Questions tests). The Color-Word Interference Test (CWIT; $M=10.9$, $S D=2.6$ ) was the only EF measure that significantly related to BMI and therefore was included in additional analyses (see Results). Based on the classic Stroop Test (Stroop, 1935), the CWIT consists of four trials that are used to assess verbal inhibition and conflict control. Subjects must first name a series of colors, followed by reading color words. Subjects then complete the inhibition trial and name the ink color (red, green, or blue) in which each color word (red, green, or blue) is printed, although these will be incongruent with one another. The final trial consists of an inhibition/switching task alternating between naming the color of the ink or reading the color word of the incongruent pairs. All four trials are measured based on the time required for the subject to complete the task. For the purposes of this study, performance on the third trial (i.e., inhibition) was assessed.

Hierarchical linear regressions were conducted to examine the effect of WASI full scale, individual WASI subtests, and D-KEFS CWIT on BMI while controlling for age, sex, and SES. Additional regression analyses were conducted using Andrew Hayes' PROCESS for SPSS model four, a mediation model (Hayes, 2013). Variables were only included in the mediation analysis if they were significantly correlated with all other variables in the model. Thus, while controlling for age, sex, and SES, performance on the D-KEFS CWIT was entered as a mediator variable on the relationship between WASI and BMI measures (Fig. 1; see Results). Following the neural analyses, post-hoc mediation analyses assessed mediation of (1) D-KEFS CWIT neural correlates on the WASIBMI relationship, (2) D-KEFS CWIT performance on the BMI-brain relationship, and (3) BMI neural correlates on the BMI-WASI relationship. Of note, while none of these 


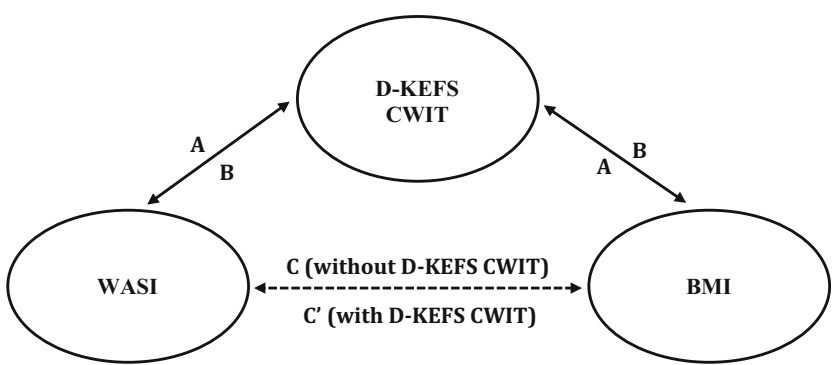

Fig. 1 Proposed model of WASI, D-KEFS CWIT, and BMI

analyses were significant, we included the third model in supplementary materials so that the reader can review the directionality of the results. All analyses were assessed with SPSS 24.0 software (IBM Corp, Somers, NY).

\section{Imaging data acquisition}

Scans were acquired using a Siemens Tim Trio 3T scanner with a 12-channel head coil. Imaging parameters for structural MRI images using a magnetization-prepared rapid gradient echo (MPRAGE) sequence were as follows: voxel size 1.0 * $1.0 * 1.0 \mathrm{~mm}^{3}$, repetition time (TR) $2,500 \mathrm{~ms}$, echo time (TE) $3.5 \mathrm{~ms}$, flip angle (FA) $8^{\circ}, 192$ slices. Imaging parameters for DTI were as follows: 64 diffusion directions, voxel size 2.0 * $2.0 * 2.0 \mathrm{~mm}^{3}$, TR 10,000 ms, TE $91 \mathrm{~ms}$, b-value 1,000 $\mathrm{s} / \mathrm{mm}^{2}, 58$ slices. Imaging parameters for the 10 -minute resting-state fMRI images using an echo-planar imaging (EPI) sequence were as follows: voxel size $3.0 * 3.0 * 3.0 \mathrm{~mm}^{3}$, TR 2,500 ms, TE $30 \mathrm{~ms}, 260$ volumes, FA $80^{\circ}, 38$ slices. Additional information regarding imaging data acquisition can be found online at http://fcon_1000.projects.nitrc.org/ fcpClassic/FcpTable.html.

\section{Surface-based morphometry}

Cortical reconstruction and volumetric segmentation was performed with the Freesurfer image analysis suite, which is documented and freely available for download online (v5.3.0; http://surfer.nmr.mgh.harvard.edu). The technical details of these procedures have been described in prior publications (Dale, Fischl, \& Sereno, 1999). Briefly, this processing includes motion correction and averaging (Reuter, Rosas, \& Fischl, 2010) of volumetric T1-weighted images, removal of non-brain tissue using a hybrid watershed/surface deformation procedure (F. Ségonne et al., 2004), automated Talairach transformation, intensity normalization (Sled, Zijdenbos, \& Evans, 1998), tessellation of the gray matter white matter boundary, automated topology correction (Fischl, Liu, \& Dale, 2001; Ségonne, Pacheco, \& Fischl, 2007), and surface deformation following intensity gradients to optimally place the gray/white and gray/cerebrospinal fluid borders at the location where the greatest shift in intensity defines the transition to the other tissue class (Dale et al., 1999; Dale \& Sereno, 1993; Fischl \& Dale, 2000). Once the cortical models are complete, a number of deformable procedures are performed for further data processing and analysis, including surface inflation (Fischl, Sereno, \& Dale, 1999), registration to a spherical atlas, which utilizes individual cortical folding patterns to match cortical geometry across subjects (Fischl, Sereno, Tootell, \& Dale, 1999), parcellation of the cerebral cortex into units based on gyral and sulcal structure (Desikan et al., 2006; Fischl et al., 2004), and creation of a variety of surface-based data, including maps of cortical volume, surface area, thickness, curvature, sulcal depth, and local gyrification index. The resulting probability maps were entered into a general linear model (GLM) evaluating regressions between all voxels and BMI, WASI, or CWIT scores, controlling for age, sex, SES, and whole-brain intracranial volume (ICV). Vertex-wise threshold was set at $p<$ 0.001. Cluster-wise threshold was corrected for at $p<0.05$ level using permutation testing with Monte Carlo simulation.

\section{Subcortical vertex analysis}

Anatomical imaging data was segmented into subcortical regions, using the FMRIB Software Library (FSL v5.0.9; Smith et al., 2004) Integrated Registration and Segmentation Tool (FIRST; https://fsl.fmrib.ox.ac.uk/fsl/fslwiki/FIRST), which is a model-based segmentation tool that allows for parcellation of several subcortical brain structures from high-resolution, T1-weighted images of the brain (Patenaude, Smith, Kennedy, \& Jenkinson, 2011; Smith et al., 2004). Importantly, this tool enables an unbiased approach to segmentation of the regions, which are notoriously hard to trace manually due to their small structure size.

Automated segmentation of 15 subcortical structures (Brain Stem and bilateral: Accumbens, Amygdala, Caudate, Hippocampus, Putamen, Pallidum, and Thalamus) was performed bby using a Bayesian probabilistic approach within FIRST. The shape and appearance models in FIRST are constructed from a library of manually segmented images. The manually generated labels are parameterized as surface meshes and then modeled as a point distribution. Using the learned models, FIRST searches through shape deformations that are linear combinations of the modes of variation to find the most probable shape instance given the observed intensities from the input image. Using T1 images, the segmentation was performed with two-stage affine transformation to standard space of MNI 152 at 1-mm resolution (Morey et al., 2009; Woolrich et al., 2009). The first stage utilized a standard 12 degrees of freedom registration to the template, and the second stage applied 12 degrees of freedom registration using an MNI152 subcortical mask to exclude voxels outside the subcortical regions. Default settings for boundary correction and number of modes (iterations) were used, which are tuned to be optimal for each structure. All segmentations were then 
visually inspected by two independent viewers to ensure satisfactory segmentation of all structures of interest. Volume also was extracted for each structure. Analyses were then performed for shape and volume to investigate differences in localized shape on a per-vertex basis and total volume of a structure with BMI, WASI, and CWIT scores. All analyses included age, sex, SES, and ICV as covariates. For shape analysis, FSL's randomize tool was used to calculate statistics from the image output after 5,000 permutations (Winkler, Ridgway, Webster, Smith, \& Nichols, 2014) and thresholdfree cluster enhancement (TFCE) with significance set at $p<$ 0.05 . Subcortical structures that passed significance were then assessed for volumetric regressions with the behavioral data.

\section{White matter analysis}

Voxelwise statistical analysis of diffusion data was carried out using tract-based spatial statistics (TBSS; Smith et al., 2006), a tool provided by FSL. First, diffusion weighted images were corrected for eddy-current-induced distortions. Fractional anisotropy (FA) images were then created by fitting a tensor model to the raw diffusion data using FSL's Diffusion Toolbox (FDT), and subsequently brain-extracted using BET (Smith, 2002). All subjects' FA data were then aligned into a common space using FMRIB's nonlinear image registration tool (FNIRT; Andersson, Jenkinson, \& Smith, 2007a, 2007b), which uses a b-spline representation of the registration warp field (Rueckert et al., 1999). Next, the mean FA image was created and thinned to produce a mean FA skeleton, which represents the centres of all tracts common to the group. Each subject's aligned FA data was then projected onto this skeleton and the resulting data fed into voxelwise cross-subject statistics. We tested for individual differences relating to BMI, WASI, and CWIT using FSL's randomize permutation-testing tool, with 5000 permutations controlling for age, sex and SES, using TFCE to correct for multiple comparisons at $p<0.05$.

\section{Functional analysis}

Resting-state fMRI data was assessed using independent components analysis (ICA), via FSL's Multivariate Exploratory Linear Optimized Decomposition into Independent Components (MELODIC) version 3.14 (Beckmann \& Smith, 2004). MELODIC separates the functional activation into a set of spatiotemporal components, which can then be analyzed as intrinsic networks of interest. Importantly, multisession temporal concatenation identifies common spatial patterns across subjects while accounting for the inconsistency of the temporal response. Using FSL, resting-state fMRI scans were motion corrected (Jenkinson, Bannister, Brady, \& Smith, 2002), brain extracted (Smith, 2002), and set through $6.0 \mathrm{~mm}$ spatial smoothing. The functional data was linearly registered to subject-specific anatomical volumes and non-linearly normalized to MNI152 standard space. After running subject-specific ICA with automatic dimensionality estimation, Automatic Removal of Motion Artifacts (AROMA) was used to remove components indicative of motion-related artifact (Pruim et al., 2015) and the normalized denoised data was then used for a group ICA analysis. The total number of output components was set to 30 , which were statistically compared with seven popular resting-state networks (Yeo et al., 2011). Twenty components were considered to represent distinct networks after showing significant spatial correlations (Pearson's $r>0.220$ ) with the reference networks.

The set of spatial maps from the group-average analysis were then used to generate subject-specific versions of the spatial maps, and associated timeseries, using dual regression (Beckmann, Mackay, Filippini, \& Smith, 2009; Filippini et al., 2009). First, for each subject, the group-average set of spatial maps was regressed (as spatial regressors in a multiple regression) into the subject's 4D space-time dataset. This resulted in a set of subject-specific timeseries, one per group-level spatial map. Next, those timeseries were regressed (as temporal regressors, again in a multiple regression) into the same 4D dataset, resulting in a set of subject-specific spatial maps, one per group-level spatial map. We then tested for individual differences relating to BMI, WASI, and CWIT using FSL's randomize permutation-testing tool, with 5,000 permutations controlling for age, sex and SES, while using TFCE to correct for multiple comparisons. Dual regression results were further corrected for number of comparisons across components and considered significant if they survived a corrected $p$ value $(p<$ 0.03 ) after weighted dependency averaging for FDR (Benjamini \& Yekutieli, 2001). Results were excluded if: 1) they were primarily localized to white matter or, 2) consisted of less than 5 voxels.

\section{Results}

\section{Behavioral results}

Intercorrelation of measures are provided in Table 2. A significant correlation was observed between WASI full scale and BMI (r[82] $=-0.30, p=0.007)$, such that increased intelligence was associated with decreased BMI. Similar negative correlations with BMI were observed for WASI similarities $(\mathrm{r}[82]=-0.38, p<0.001)$ and WASI vocabulary $(\mathrm{r}[82]=$ $-0.30, p=0.007)$ subtests. WASI block design $(\mathrm{r}[82]=$ $-0.20, p=0.07)$ and WASI matrix reasoning $(\mathrm{r}[82]=-0.05$, $p=0.63$ ) subtests were not significantly correlated with BMI. Furthermore, D-KEFS CWIT, but not other subscales of the D-KEFS, was significantly related to BMI ( $[$ [82] $=-0.29, p=$ $0.008)$, as increased inhibitory control was associated with decreased BMI. D-KEFS CWIT also was significantly positively related to WASI full scale $(\mathrm{r}[82]=0.32, p=0.003)$. 
Table 2 Summary of Pearson correlations

\begin{tabular}{|c|c|c|c|c|c|c|}
\hline Measure & 1 & 2 & 3 & 4 & 5 & 6 \\
\hline 1. WASI full scale & - & & & & & \\
\hline 2. WASI similarities & $0.84^{\dagger}$ & - & & & & \\
\hline 3. WASI vocabulary & $0.80^{\dagger}$ & $0.69^{\dagger}$ & - & & & \\
\hline 4. WASI block design & $0.80^{\dagger}$ & $0.56^{\dagger}$ & $0.42^{\dagger}$ & - & & \\
\hline 5. WASI matrix reasoning & $0.75^{\dagger}$ & $0.48^{\dagger}$ & $0.39^{\dagger}$ & $0.61^{\dagger}$ & - & \\
\hline 6. D-KEFS CWIT & $0.32^{\S}$ & 0.17 & $0.29^{\S}$ & $0.31^{\S}$ & $0.24 *$ & - \\
\hline 7. BMI & $-0.30^{\S}$ & $-0.38^{\dagger}$ & $-0.30^{\S}$ & -0.20 & -0.05 & $-0.29^{\S}$ \\
\hline
\end{tabular}

$\mathrm{N}=82,{ }^{*} p<0.05,{ }^{\S} p<0.01,{ }^{\dagger} p<0.001$

Similar relationships with D-KEFS CWIT were observed for WASI vocabulary $(\mathrm{r}[82]=0.29, p=0.009)$, WASI block design $(\mathrm{r}[82]=0.31, p=0.005)$, and WASI matrix reasoning $(\mathrm{r}[82]=0.24, p=0.03)$ subtests. Subsequently, because of the relationships between WASI, CWIT and BMI, we assessed the amount of variance explained by WASI on BMI, WASI on CWIT, and CWIT on BMI, while controlling for factors (i.e., age, sex, SES) that have shown to contribute to the relationships between intelligence, BMI, and EF.

Using hierarchical linear regressions [controlling for age, sex, and SES], greater WASI full scale was associated with lower BMI $\left(\Delta R^{2}=0.08, p=0.007\right)$. Additional regressions with WASI subtests of similarities $\left(\Delta R^{2}=0.12, p=0.001\right)$ and vocabulary $\left(\Delta R^{2}=0.09, p=0.004\right)$ each contributed significant variance to the estimation of BMI. Greater D-KEFS CWIT performance was also associated with lower BMI $\left(\Delta R^{2}=0.11, p=0.001\right)$.

Consistent with specifications for mediation analyses (Hayes, 2013), variables were included in analyses if they were significantly correlated with all other variables in the model. Thus, mediation analyses were conducted with the WASI full scale, WASI vocabulary, and BMI, including the D-KEFS CWIT as a mediator (Table 3). A significant effect was observed for WASI full scale on D-KEFS CWIT (a; $b=$ $0.07, t=3.21, p=0.002)$ and D-KEFS CWIT on BMI ( $\mathrm{b} ; b=$ $-0.55, t=-2.57, p=0.012)$ such that the indirect effect $(\mathrm{a} * \mathrm{~b})$ of D-KEFS CWIT on the relation between WASI full scale and BMI was significant $(b=-0.04,95 \%$ BC boot-strap CI -0.09 to -0.01$)$. The significant effect of WASI full scale on BMI $(b=-0.12, t=-2.78, p=0.007)$ became nonstatistically significant $(b=-0.08, t=-1.82, p=0.073)$ in the presence of the indirect effect of D-KEFS CWIT (95\% BC boot-strap CI -0.17 to 0.01 ). This pattern of results demonstrates that inhibitory control fully mediated the relation between intelligence and BMI.

An additional analysis was performed for the WASI vocabulary subtest. A significant effect was observed for WASI vocabulary on D-KEFS CWIT (a; $b=0.09, t=2.76, p=$ $0.007)$ and D-KEFS CWIT on BMI ( $\mathrm{b} ; b=-0.55, t=-2.63$, $p=0.011)$ such that the indirect effect $(\mathrm{a} * \mathrm{~b})$ of D-KEFS
CWIT on the relation between WASI vocabulary and BMI was significant $(b=-0.05,95 \% \mathrm{BC}$ boot-strap $\mathrm{CI}-0.10$ to $-0.02)$. The significant effect of WASI vocabulary on BMI ( $b$ $=-0.18, t=-2.99, p=0.004)$ remained statistically significant $(b=-0.13, t=-2.17, p=0.033)$ in the presence of the indirect effect of D-KEFS CWIT (95\% BC boot-strap CI -0.25 to $-0.01)$. This pattern of results demonstrates that EF partially mediated the relation between the WASI vocabulary subtest and BMI.

Due to the use of cross-sectional data, setting BMI as the independent variable and WASI full scale as the dependent variable produced identical results. The significant effect of BMI on WASI full scale $(b=-0.74, t=-2.78, p=0.007$ ) became nonstatistically significant $(b=-0.51, t=-1.82, p=$ 0.073 ) in the presence of the indirect effect of D-KEFS CWIT (95\% BC boot-strap CI -1.06 to 0.05 ), whereas the significant effect of BMI on WASI vocabulary $(b=-0.58, t=-2.99, p=$ 0.004 ) remained significant $(b=-0.44, t=-2.17, p=0.033)$ in the presence of the indirect effect of D-KEFS CWIT $(95 \%$ BC boot-strap CI -0.84 to -0.04 ).

\section{Body mass index results}

Regressions with cortical grey matter morphometry indicated that increased body mass index (BMI) significantly associated with decreased cortical thickness in the left ACC and part of the left vmPFC (Fig. 2a; $p<0.008$ ). Furthermore, increased BMI related to significant changes in subcortical morphometry, including shape changes and increased volume of the left globus pallidus (Fig. 2b; $t=$ 3.23, $p=0.002$ ) and right amygdala (Fig. $2 \mathrm{~b} ; t=2.93, p=$ 0.004). Examination of indicators of white matter integrity (FA) further revealed that increased BMI related to increased FA in the left uncinate fasciculus (Fig. 2c; $p<$ 0.008 ), suggesting increased body mass related to increased structural integrity within this tract. Body mass index did not show a significant association with any of the intrinsic connectivity networks identified from restingstate functional connectivity. 
Table 3 Summary of mediation results

\begin{tabular}{|c|c|c|c|c|c|c|c|c|}
\hline & $\begin{array}{l}\text { Independent } \\
\text { variable (IV) }\end{array}$ & Mediator (M) & $\begin{array}{l}\text { Dependent } \\
\text { variable (DV) }\end{array}$ & $\begin{array}{l}\text { Effect of } \\
\text { IV on M (a) }\end{array}$ & $\begin{array}{l}\text { Effect of M } \\
\text { on DV (b) }\end{array}$ & $\begin{array}{l}\text { Indirect effect } \\
(\mathrm{a} \times \mathrm{b})\end{array}$ & $\begin{array}{l}\text { Total } \\
\text { effect (c) }\end{array}$ & $\begin{array}{l}\text { Direct } \\
\text { effect (c') }\end{array}$ \\
\hline 1 & WASI Full Scale & D-KEFS CWIT Inhibition & BMI & 0.07 & -0.55 & -0.04 & -0.12 & $-0.08^{*}$ \\
\hline 2 & WASI Vocabulary & D-KEFS CWIT Inhibition & BMI & 0.09 & -0.55 & -0.05 & -0.18 & -0.13 \\
\hline 3 & BMI & D-KEFS CWIT Inhibition & WASI Full Scale & -0.19 & 1.27 & -0.24 & -0.74 & $-0.51^{*}$ \\
\hline 4 & BMI & D-KEFS CWIT Inhibition & WASI Vocabulary & -0.19 & 0.72 & -0.14 & -0.58 & -0.44 \\
\hline
\end{tabular}

Beta values are displayed for each significant effect $(p<0.05)$, unless where $*$ for the direct effect indicates full mediation $(p>0.05)$

\section{Wechsler abbreviated scale of intelligence, second edition (WASI-II)}

Cortical grey matter morphometry results indicated that increased scores on intelligence tests related primarily to increased thickness and volume of parietal regions (Fig. 3). Increased WASI full scale scores associated with increased volume in the left superior parietal lobule (SPL) (Fig. 3a; $p$ $<0.005$ ). Likewise, analysis of subtest scores (Fig. 3b) revealed increased performance on the similarities subtest also related to increased thickness $(p=0.001)$ and volume $(p=$ $0.006)$ in the left SPL, as well as increased thickness in the right inferior parietal lobule (IPL) $(p<0.01)$ and increased volume in the right visual cortex $(p<0.02)$. Additionally, increased vocabulary subtest scores associated with increased volume in the right fusiform gyrus $(p<0.03)$.
To assess functional attributes related to performance on the WASI, we examined resting-state functional connectivity using ICA. Results indicated that higher intelligence scores significantly related to increased connectivity of a visual ICN with the dorsolateral PFC, lingual gyrus, cuneus, and precuneus (Fig. $3 c ; p<0.03$ ). This same finding for the precuneus also was found for the similarities subtest $(p<$ 0.03). Performance on the WASI full scale and subtest scores did not show significant associations with subcortical structure or white matter integrity.

\section{D-KEFS color-word interference test}

D-KEFS Color-Word Interference Test (CWIT) performance was significantly related to cortical thickness in frontal and parietal regions (Fig. 4a), such that increased inhibitory

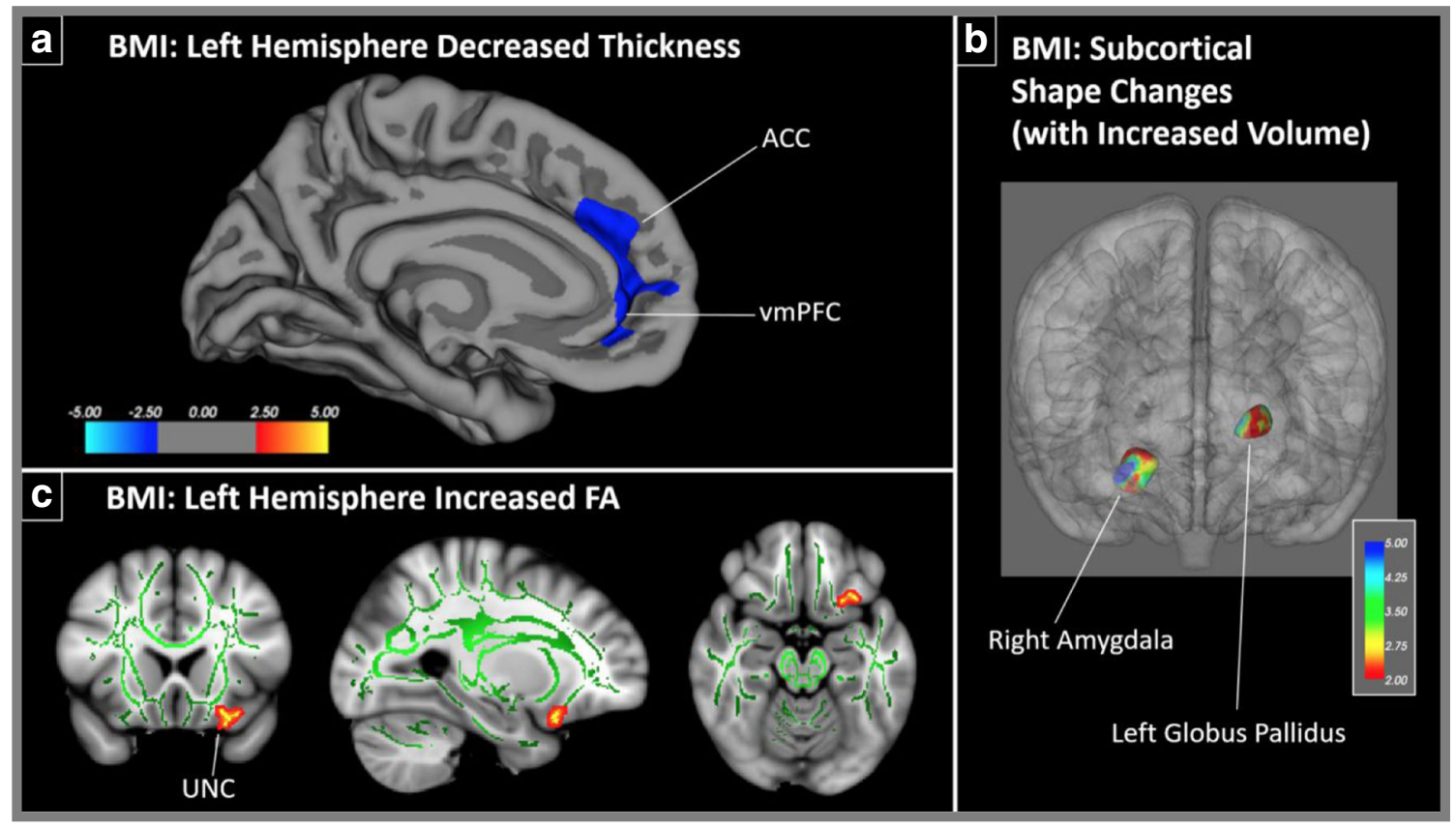

Fig. 2 a Increased BMI associated with decreased cortical thickness in left hemisphere frontal regions including the anterior cingulate cortex (ACC) and ventromedial prefrontal cortex (vmPFC). Color bar represents the significance as $-\log (10) p$. b Increased BMI relates to differences in shape and increased volume for the left globus pallidus and right amygdala. Color bar represent increasing statistical significance (from red to blue) of the $F$-statistic. c Provided from left to right are coronal, sagittal, and axial views of a standard anatomical brain image. The average white matter skeleton for all subjects of the present study is shown in green, with the highlighted red area in the left hemisphere indicating a significant increase of FA in the uncinate fasciculus (UNC) with increased BMI 


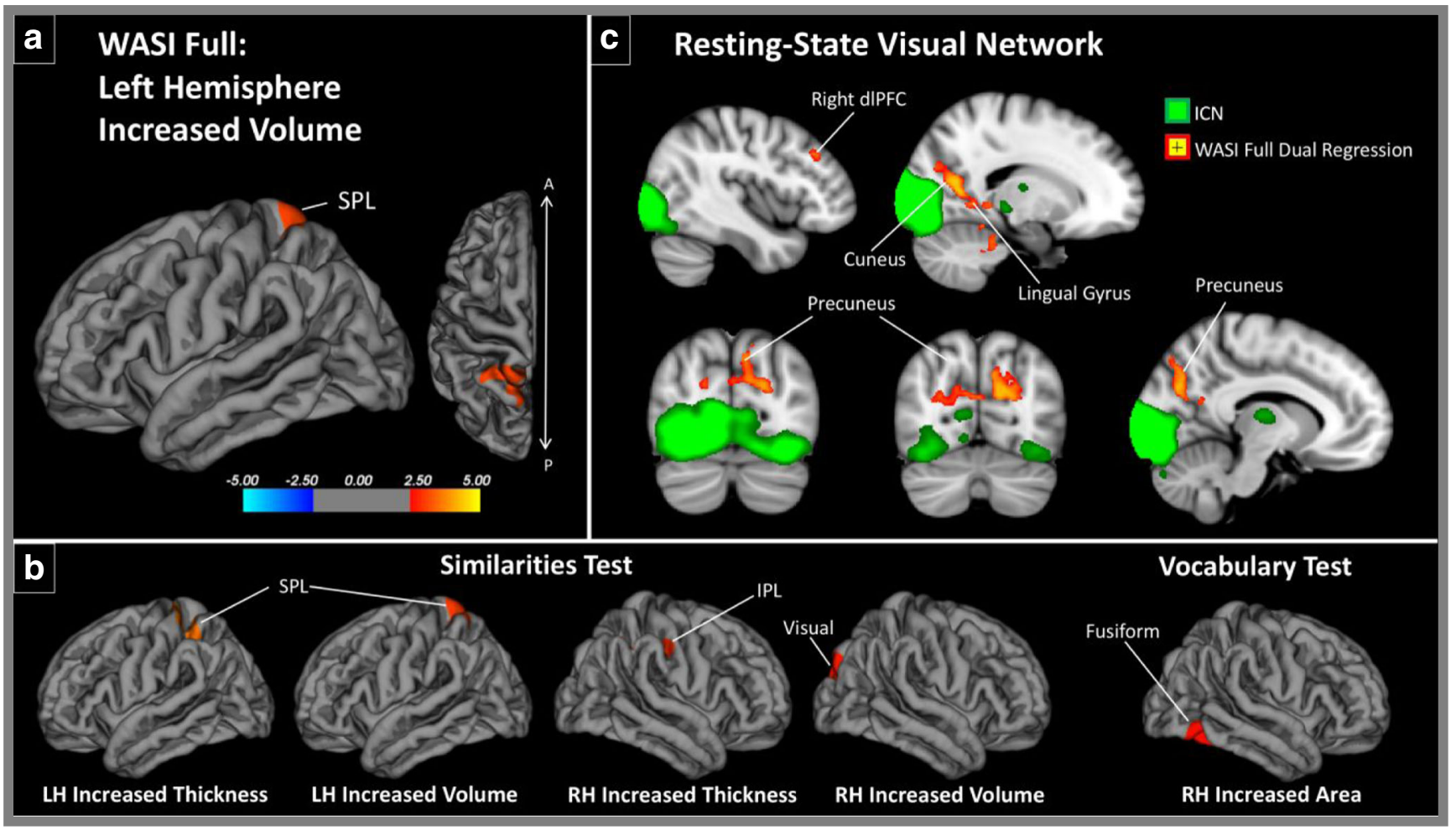

Fig. 3 a Increased intelligence (WASI full scale) related to increased volume in the left superior parietal lobule (SPL). b Increased performance on the similarities test were associated with increased thickness and volume in the left SPL, as well as increased thickness in the right inferior parietal lobule (IPL) and increased volume in the right visual cortex, whereas increased vocabulary scores were associated with increased area in the right fusiform gyrus. c Dual regression results indicated that increased WASI performance showed increased representation of a visual ICN, which specifically related to increased connectivity with the right dorsolateral PFC, lingual gyrus, cuneus, and precuneus control ability associated with increased thickness in the left SPL $(p<0.007)$ and left lateral orbitofrontal cortex (OFC) $(p<$ $0.03)$. Subcortical morphometry results indicated that increased performance on the D-KEFS CWIT related to a significant change in shape and increased volume of the left nucleus accumbens (Fig. 4b; $t=2.94, p=0.004$ ), suggesting that increased inhibitory control was associated with increased size of the left nucleus accumbens. D-KEFS CWIT performance did not show any significant associations with FA measures of white matter structural integrity.

Resting-state ICA analyses further revealed that increased performance on the D-KEFS CWIT associated with increased functional connectivity of the default mode network (DMN) with bilateral insular and opercular regions, as well as the right amygdala and hippocampus (Fig. 4c; $p<0.03$ ). This suggested that increased inhibition related to increased acrossnetwork connectivity of the DMN.

\section{Discussion}

This study was the first, to our knowledge, to investigate the behavioral and underlying neural connections among BMI, intelligence, and $\mathrm{EF}$ via a comprehensive examination of cortical and subcortical morphometry, white matter structural integrity, and resting-state functional connectivity. Behaviorally, BMI was negatively correlated with both intelligence and EF abilities, such that increased BMI associated with decreased WASI and D-KEFS CWIT performance above and beyond the effects of age, sex, and SES. Additional mediation analyses further revealed that inhibitory control, as measured by the D-KEFS CWIT, mediated the negative relationship between BMI and intelligence. Given these associations, we suspected that a neural analysis could help to clarify such an interconnected behavioral relationship. All three measures displayed unique associations with structural or functional neural correlates. However, their relationship with one another is best understood when considering cortical morphometry, because this was the only metric that significantly associated with each measure. Unfortunately, there was no distinct overlap in regional morphometric changes across BMI, intelligence, and D-KEFS CWIT. Nonetheless, these morphometric results reveal shared structural associations among intelligence and inhibitory control and that increased BMI relates to thinning of medial prefrontal regions that have routinely associated with cognitive/ emotional control ability. Collectively, our multimodal analyses further indicate several structural and functional components that track changes in each of these measures independently. 

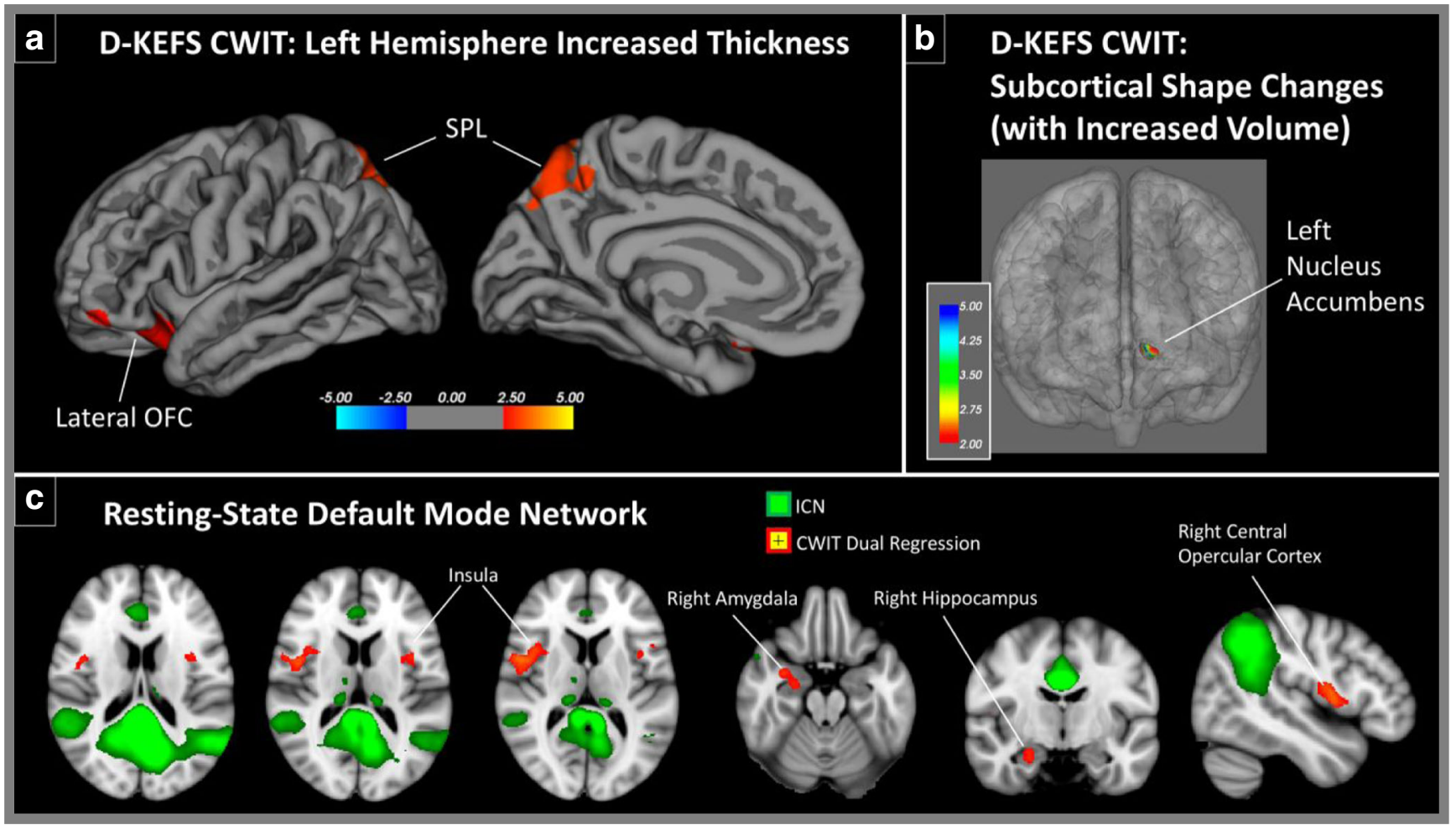

Fig. 4 a Increased inhibitory control, as measured by the D-KEFS CWIT, associated with increased thickness in the left superior parietal lobule (SPL) and the left-lateral orbitofrontal cortex (OFC). b Increased CWIT scores also related to significant shape changes and increased volume for the left nucleus accumbens. $\mathbf{c}$ Dual regression results indicating increased D-KEFS CWIT scores related to increased connectivity of the default mode network with bilateral insular and central-opercular regions, as well as the right amygdala and hippocampus

\section{Body mass index, executive function, and intelligence}

The behavioral results support our hypothesis that a relationship between BMI and intelligence is mediated by EF ability, namely inhibitory control. These findings align with research demonstrating differences in cognitive ability accompanying higher BMI or obesity (Kanazawa, 2014; Lavagnino, Arnone, Cao, Soares, \& Selvaraj, 2016). Moreover, the D-KEFS CWIT was the only available D-KEFS measure that significantly correlated with BMI, in line with previous studies showing that reduced inhibitory control is a risk factor for weight gain due to a relationship with unhealthy eating habits and heightened impulsivity (Jasinska et al., 2012). Additionally, decreased activation of frontal inhibitory control regions, as an index of control over response inhibition, also is predictive of elevated weight (Batterink, Yokum, \& Stice, 2010), and a recent study suggests that a relationship between increased BMI and decreased cortical thickness in the right superior frontal gyrus is mediated by reduced inhibitory control (Lavagnino et al., 2016). Importantly, we extend these findings by showing that inhibitory control also mediates a negative association of BMI with general intelligence.

In regard to the neural results, we found cortical morphometry to be the only metric significantly related to each behavioral measure. Interestingly, however, we did not find overlap in these morphometric findings. Intelligence and D-KEFS CWIT performance were associated with increased thickness and volume across frontal and parietal regions, namely in the SPL. In contrast, increased BMI related to reduced cortical thickness in dorsal/anterior ACC extending into the vmPFC, in accordance with previous studies documenting similar associations of BMI with cortical structure in prefrontal regions (Hassenstab et al., 2012; Kennedy et al., 2016; Medic et al., 2016). Of note, post-hoc analyses revealed that average cortical thickness in this region showed a positive correlation with WASI and D-KEFS CWIT performance. Although these correlations were not statistically significant, their directionality bolsters our interpretation of contrasting associations of cortical morphometry with BMI compared with intelligence and EF ability (see Supplementary Materials). However, given a lack of overlap in the neural results, we are careful to relate directly these structural correlates to the observed behavioral mediation. Our findings nonetheless highlight two key associations to take into consideration when evaluating this mediation. First, we found support for similar morphometric associations of intelligence and inhibitory control with superior parietal regions, which may explain the close behavioral associations between these measures, as well as why both ultimately show a similar relationship with BMI. The restingstate ICA results further corroborate the association between 
intelligence and inhibitory control. Increased intelligence correlated with increased connectivity of a visual network primarily with frontal and occipital regions, supporting the notion that higher intelligence is driven by across-network connectivity of the lateral PFC in order to facilitate global multisystem interactions in the brain (Cole, Ito, \& Braver, 2015). Likewise, inhibitory control ability related to increased resting-state connectivity of the default mode network with nodes of the salience and cingulo-opercular networks that underlie the integration of sensory and affective information (Coste \& Kleinschmidt, 2016; Power et al., 2011; Seeley et al., 2007). Recent models propose that the salience and cingulo-opercular networks coordinate switching between the default mode "task-negative" and frontoparietal/central executive "task-positive" networks, effectively signaling reduced DMN activity when capturing salient information in need of further processing and attention (Bressler \& Menon, 2010; Cocchi, Zalesky, Fornito, \& Mattingley, 2013; Goulden et al., 2014; Jilka et al., 2014; Sridharan, Levitin, \& Menon, 2008). The present findings therefore add to a growing consensus that adaptive control ability relies on intricate connections among frontoparietal, default, and salience/cinguloopercular networks, whereby the specific components of these networks engage with cognitive measures, such as inhibitory control or intelligence to differing degrees.

Second, the relationship between increased BMI and widespread cortical thinning in the ACC and vmPFC suggests that increased BMI has an extensive relationship with brain structure, which may have corresponding functional and behavioral consequences, considering that these frontal cortical regions are highly involved in both decisionmaking (Kennerley \& Walton, 2011) and inhibitory control (Li \& Sinha, 2008; Munakata et al., 2011), especially when reward or emotion is salient. The ventral prefrontal cortex and its connections with limbic structures has consistently shown an association with these "hot" motivationally significant EFs (Zelazo \& Cunningham, 2007). The dorsal ACC also is considered as an important hub for networks that engage in inhibitory control, increasing connectivity with other top-down control regions as the demand for inhibitory control increases (Spielberg, Miller, Heller, \& Banich, 2015). Moreover, these medial prefrontal regions are integrated with intelligence networks proposed by PFIT (Colom et al., 2009; Jung \& Haier, 2007; Vakhtin et al., 2014) and intellectual ability relies on inhibitory control to suppress task-irrelevant information (Dempster, 1991), possibly suggesting why BMI, inhibitory control, and intelligence share such an interconnected relationship. Cortical thinning of the medial PFC may disrupt the P-FIT and EF networks, such that the observed structural associations with BMI ultimately has functional consequences when these medial prefrontal regions are recruited for cognitively demanding tasks.
Body mass index and frontolimbic structural changes

The use of a comprehensive, multimodal neural analysis yielded interesting results beyond the original focus of this neurocognitive investigation. In particular, these findings show that increased BMI associates with morphometric alterations in a network consisting of the ACC, uncinate fasciculus (UF), and amygdala. Although there were hemispheric differences in these findings-left ACC and left UF in contrast to the right amygdala - these three structures nonetheless comprise a frontolimbic network that is involved in regulating emotion and reward processing (Botvinick, Cohen, \& Carter, 2004; Bush, Luu, \& Posner, 2000; Etkin, Egner, Peraza, Kandel, \& Hirsch, 2006; Ghashghaei, Hilgetag, \& Barbas, 2007; Petrides \& Pandya, 2007; Wang et al., 2009). More specifically, the ACC and subgenual regions that are considered the VmPFC send projections to the amygdala through the UF, as well as other white matter tracts. These projections are considered to form control afferents that ultimately provide inhibitory control over emotional response (Depue, Orr, Smolker, Naaz, \& Banich, 2016).

The observed positive relationship between FA in the UF and BMI was surprising, considering that previous studies generally find negative associations between white matter structural integrity and measures of obesity. However, indicators of increased weight have previously associated with increased white matter integrity or volume in several regions (Birdsill et al., 2017; Yokum et al., 2012). Moreover, higher FA in the UF has been found to correlate with sensitivity to punishment and reward-related activity in the nucleus accumbens (Camara, Rodriguez-Fornells, \& Munte, 2010), further implicating changes in this tract with higher BMI as representative of differences in emotion and reward processing.

A closer look at the subcortical findings also suggests the possibility that higher BMI may be associated with differences in the initial processing of salient information related to emotion or reward. The amygdala detects saliency in the environment (Adolphs, 2010; Cunningham \& Brosch, 2012; Gonzalez Andino \& Grave de Peralta Menendez, 2012), which may be heightened in people with higher BMIs, inducing food driven behavior. Furthermore, our finding of increased size and changes in shape of the globus pallidus with increased BMI may ultimately reflect effects on gating mechanisms signaling reward to higher-level cognitive control (Haber \& Knutson, 2010; Hong \& Hikosaka, 2008), perhaps also corresponding to the observed behavioral association between BMI and inhibitory control. Similar to our finding for increased FA in the UF, increased volume in the amygdala and globus pallidus might reflect hyperactive reward or emotion systems, although we are careful to infer a direct relationship between size and function. Nonetheless, our results do support an association of BMI with changes in the overall structural architecture of these subcortical structures. 
Supporting these frontolimbic findings, increased BMI has been linked with impulsivity and a variety of emotional influences on eating behavior. Previous research shows that BMI and emotion-driven impulsivity are positively linked among adolescents, such that increases in BMI predict higher levels of both positive and negative urgency (Delgado-Rico, RíoValle, González-Jiménez, Campoy, \& Verdejo-García, 2012). Furthermore, higher BMI is positively linked to appetitive behaviors that include food responsiveness and emotional overeating (Hunot et al., 2016). Overweight and obese individuals also are more likely to represent emotion- and stressrelated eating habits (Ozier et al., 2008), as well as overeat during emotional periods (Davis, Levitan, Muglia, Bewell, \& Kennedy, 2004). Alterations in a regulatory frontolimbic network that incorporates the ACC, UF, and amygdala, as well as changes to additional subcortical regions, such as the globus pallidus, may ultimately help to explain these differences in behavior. This proposal is supported by a similar model from Cyders \& Smith (2008), which suggests that disruptions to the ventral prefrontal cortex and amygdala connectivity may ultimately facilitate the development of urgency traits. Additionally, Kennedy, Collins, \& Luciana (2016) recently showed that higher BMI in adolescents is associated with widespread structural alterations in medial PFC and subcortical regions that associate with reward processing. Although differences in reward and emotion processing with higher BMI might exist separately from differences in inhibitory control or intelligence, we speculate that heightened sensitivity to salient information might nonetheless interfere with efficient engagement of top-down control networks. Moreover, our findings of widespread thinning in medial PFC and accompanying changes to white matter and subcortical regions suggests alterations in cortico-striatal-thalamo-cortical loops, specifically the anterior cingulate loop, which has been noted to connect the ACC with the amygdala/hippocampus and ventral pallidum (Alexander, DeLong, \& Strick, 1986).

\section{Limitations}

Although results of the current study form an integrated picture of brain-mind-body associations, there are several limitations to address. Body mass index is an indirect measure of internal body physiology and therefore cannot be used as a direct assessment of physical health. For example, BMI can sometimes inaccurately classify highly athletic individuals who are in peak physical health as overweight or obese (Prentice \& Jebb, 2001). However, although BMI does not necessarily indicate a particular health state for an individual, it does provide an efficient method for predicting the presence or risk of developing specific diseases that accompany increased fat or stress on the body. Body mass index correlates better with visceral fat area and insulin sensitivity compared with body adiposity index (BAI), another popular indirect measure of body fat (Hung et al., 2012; Suchanek et al., 2012). Body mass index also shows positive correlations with cholesterol and blood pressure, emphasizing the importance of this measurement in assessing risk for the development of serious health complications (McAdams, Van Dam, \& Hu, 2007). Furthermore, we used a range of BMI values to assess behavioral and neural relationships with increased or decreased BMI, as opposed to grouping subjects into distinct groups that may not be entirely accurate for each subject. Thus, we argue that BMI remains an appropriate measure for assessing cognitive and neural relationships with body physiology. Nonetheless, future studies pursuing a brainmind-body approach can certainly benefit from using additional physiological measures that make more direct assessments of physical health.

To assess indicators of white matter tract integrity in relation to our variables of interest we used FSL's TBSS analysis, which is well-suited for minimizing partial volume effects and restricts analyses to a skeletonized version of the group average FA map. We also utilized TFCE, which avoids setting arbitrary cluster-forming thresholds (Stephen M. Smith \& Nichols, 2009), and nonparametric permutation testing to correct for multiple comparisons (Nichols \& Holmes, 2001). Thus, the exclusion of peripheral tract integrity and use of more computationally expensive statistical analyses may have obscured some additional relationships between white matter and BMI, intelligence, or EF. Additionally, as with all analyses, we controlled for relevant demographic factors that may have further restricted the findings and specifically assessed correlational relationships as opposed to placing individuals in groups.

Surprisingly, there were few functional results from the resting-state data that related to the structural findings, suggesting that task-based fMRI during an intelligence or EF task may be needed to clarify the observed grey and white matter structural regressions. Furthermore, we were unable to identify the causal nature of the relationships presented here, given the use of a cross-sectional dataset. Thus, we can only demonstrate that these variables are related. Given that age, sex, and SES are important covariates to consider, future studies can examine whether similar relationships are found in more specific populations, such as those with a smaller age range.

\section{Conclusions}

Collectively, our results support a negative association of BMI with frontal cortical morphometry that contrasts the strengthened structural and functional networks associated with intelligence and EF. Although we did not identify a particular neural feature underlying the mediating effect of inhibitory control on BMI and intelligence, our findings do highlight important regions to consider when assessing such a 
relationship. This primarily includes regions comprising frontolimbic and frontoparietal networks important for affective regulation and cognitive control ability, respectively. Because we only assessed inherent structural and functional architecture, studies that use experimental paradigms to examine these relationships directly will help to clarify our results. As well, a longitudinal examination of these relationships to assess causality also remains a compelling and complex area for future research.

Publisher's note Springer Nature remains neutral with regard to jurisdictional claims in published maps and institutional affiliations.

\section{References}

Adolphs, R. (2010). What does the amygdala contribute to social cognition? Annals of the New York Academy of Sciences. https://doi.org/ 10.1111/j.1749-6632.2010.05445.x

Alexander, G. E., DeLong, M. R., \& Strick, P. L. (1986). Parallel organization of functionally segregated circuits linking basal ganglia and cortex. Annual Review of Neuroscience. https://doi.org/10.1146/ annurev.neuro.9.1.357

Andersson, J. L. R., Jenkinson, M., \& Smith, S. (2007a). Non-linear registration, aka spatial normalisation. FMRIB Technial Report TR07JA2. Oxford Centre for Functional Magnetic Resonance Imaging of the Brain, Department of Clinical Neurology, Oxford University, Oxford, UK.

Andersson, J. L. R., Jenkinson, M., \& Smith, S. M. (2007b). Non-linear optimisation. FMRIB technical report TR07JA1. Retrieved from http://fsl.fmrib.ox.ac.uk/analysis/techrep/tr07ja1/tr07ja1.pdf

Au, J., Sheehan, E., Tsai, N., Duncan, G. J., Buschkuehl, M., \& Jaeggi, S. M. (2015). Meta-analysis, improving fluid intelligence with training on working memory. Psychonomic Bulletin \& Review, 22, 366-377. https://doi.org/10.1016/j.cognition.2008.05.007

Ball, K., \& Crawford, D. (2005). Socioeconomic status and weight change in adults: A review. Social Science and Medicine, 60(9), 1987-2010. https://doi.org/10.1016/j.socscimed.2004.08.056

Barbey, A. K., Colom, R., Solomon, J., Krueger, F., Forbes, C., \& Grafman, J. (2012). An integrative architecture for general intelligence and executive function revealed by lesion mapping. Brain, 135(4), 1154-1164. https://doi.org/10.1093/brain/aws021

Batterink, L., Yokum, S., \& Stice, E. (2010). Body mass correlates inversely with inhibitory control in response to food among adolescent girls: An fMRI study. NeuroImage, 52(4), 1696-1703. https://doi. org/10.1016/j.neuroimage.2010.05.059

Beckmann, C. F., \& Smith, S. M. (2004). Probabilistic Independent Component Analysis for Functional Magnetic Resonance Imaging. IEEE Transactions on Medical Imaging, 23(2), 137-152. https:// doi.org/10.1109/TMI.2003.822821

Beckmann, C., Mackay, C., Filippini, N., \& Smith, S. (2009). Group comparison of resting-state FMRI data using multi-subject ICA and dual regression. NeuroImage, 47, S148. https://doi.org/10. 1016/S1053-8119(09)71511-3

Benjamini, Y., \& Yekutieli, D. (2001). The control of the false discovery rate in multiple testing under dependency. Annals of Statistics, 1165-1188. https://doi.org/10.1214/aos/1013699998

Birdsill, A. C., Oleson, S., Kaur, S., Pasha, E., Ireton, A., Tanaka, H., \& Haley, A. (2017). Abdominal obesity and white matter microstructure in midlife. Human Brain Mapping. https://doi.org/10.1002/ hbm. 23576
Botvinick, M. M., Cohen, J. D., \& Carter, C. S. (2004). Conflict monitoring and anterior cingulate cortex: An update. Trends in Cognitive Sciences. https://doi.org/10.1016/j.tics.2004.10.003

Boyle, C. P., Raji, C. A., Erickson, K. I., Lopez, O. L., Becker, J. T., Gach, H. M., ... Thompson, P. M. (2015). Physical activity, body mass index, and brain atrophy in Alzheimer's disease. Neurobiology of Aging, 36(S1), S194-S202. https://doi.org/10.1016/j. neurobiolaging.2014.05.036

Bressler, S. L., \& Menon, V. (2010). Large-scale brain networks in cognition: emerging methods and principles. Trends in Cognitive Sciences, 14(6), 277-290. https://doi.org/10.1016/j.tics.2010.04. 004

Bush, G., Luu, P., \& Posner, M. (2000). Cognitive and emotional influences in anterior cingulate cortex. Trends in Cognitive Sciences, 4(6), 215-222. https://doi.org/10.1016/S1364-6613(00)01483-2

Camara, E., Rodriguez-Fornells, A., \& Munte, T. F. (2010). Microstructural brain differences predict functional hemodynamic responses in a reward processing task. Journal of Neuroscience. https://doi.org/10.1523/JNEUROSCI.0111-10.2010

Cocchi, L., Zalesky, A., Fornito, A., \& Mattingley, J. B. (2013). Dynamic cooperation and competition between brain systems during cognitive control. Trends in Cognitive Sciences, 17(10), 493-501. https:// doi.org/10.1016/j.tics.2013.08.006

Cohen, S., \& Herbert, T. B. (1996). Health psychology: Psychological factors and physical disease from the perspective of human psychoneuroimmunology. Annual Review of Psychology, 47, 113-142. https://doi.org/10.1146/annurev.psych.47.1.113

Cole, M. W., Ito, T., \& Braver, T. S. (2015). Lateral prefrontal cortex contributes to fluid intelligence through multinetwork connectivity. Brain Connectivity, 5(8), 497-504. https://doi.org/10.1089/brain. 2015.0357

Cole, M. W., Reynolds, J. R., Power, J. D., Repovs, G., Anticevic, A., \& Braver, T. S. (2013). Multi-task connectivity reveals flexible hubs for adaptive task control. Nature Neuroscience, 16(9), 1348-1355. https://doi.org/10.1038/nn.3470

Cole, M. W., Yarkoni, T., Repovs, G., Anticevic, A., \& Braver, T. S. (2012). Global connectivity of prefrontal cortex predicts cognitive control and intelligence. Journal of Neuroscience, 32(26), 89888999. https://doi.org/10.1523/JNEUROSCI.0536-12.2012

Colom, R., Haier, R. J., Head, K., Álvarez-Linera, J., Quiroga, M. Á., Shih, P. C., \& Jung, R. E. (2009). Gray matter correlates of fluid, crystallized, and spatial intelligence: Testing the P-FIT model. Intelligence, 37(2), 124-135. https://doi.org/10.1016/j.intell.2008. 07.007

Cotman, C. W., \& Berchtold, N. C. (2002). Exercise: A behavioral intervention to enhance brain health and plasticity. Trends in Neurosciences. https://doi.org/10.1016/S0166-2236(02)02143-4

Cotman, C. W., Berchtold, N. C., \& Christie, L. A. (2007). Exercise builds brain health: key roles of growth factor cascades and inflammation. Trends in Neurosciences. https://doi.org/10.1016/j.tins. 2007.06.011

Cunningham, W. a., \& Brosch, T. (2012). Motivational salience: Amygdala tuning from traits, needs, values, and goals. Current Directions in Psychological Science, 21(1), 54-59. https://doi.org/ 10.1177/0963721411430832

Cyders, M. A., \& Smith, G. T. (2008). Emotion-based dispositions to rash action: Positive and negative urgency. Psychological Bulletin, 134(6), 807-828. https://doi.org/10.1037/a0013341

Dale, A. M., Fischl, B., \& Sereno, M. I. (1999). Cortical surface-based analysis: I. Segmentation and surface reconstruction. NeuroImage, 9(2), 179-194. https://doi.org/10.1006/nimg.1998.0395

Dale, A. M., \& Sereno, M. I. (1993). Improved localizadon of cortical activity by combining EEG and MEG with MRI cortical surface reconstruction: A linear approach. Journal of Cognitive Neuroscience, 5(2), 162-176. https://doi.org/10.1162/jocn.1993.5. 2.162 
Davis, C., Levitan, R. D., Muglia, P., Bewell, C., \& Kennedy, J. L. (2004). Decision-making deficits and overeating: A risk model for obesity. Obesity Research, 12(6), 929-935. https://doi.org/10.1038/oby. 2004.113

Delgado-Rico, E., Río-Valle, J. S., González-Jiménez, E., Campoy, C., \& Verdejo-García, A. (2012). BMI predicts emotion-driven impulsivity and cognitive inflexibility in adolescents with excess weight. Obesity, 20(8), 1604-1610. https://doi.org/10.1038/oby.2012.47

Delis, D., Kaplan, E., \& Kramer, J. (2001). Delis-Kaplan executive function system (D-KEFS). Canadian Journal of School Psychology, 20(1-2), 117-128. https://doi.org/10.1177/0829573506295469

Dempster, F. N. (1991). Inhibitory processes: A negleted dimension of intelligence. Intelligence, 15(2), 157-173. https://doi.org/10.1016/ 0160-2896(91)90028-C

Depue, B. E., Orr, J. M., Smolker, H. R., Naaz, F., \& Banich, M. T. (2016). The organization of right prefrontal networks reveals common mechanisms of inhibitory regulation across cognitive, emotional, and motor processes. Cerebral Cortex, 26(4), 1634-1646. https:// doi.org/10.1093/cercor/bhu324

Desikan, R. S., Ségonne, F., Fischl, B., Quinn, B. T., Dickerson, B. C., Blacker, D., ... Killiany, R. J. (2006). An automated labeling system for subdividing the human cerebral cortex on MRI scans into gyral based regions of interest. NeuroImage, 31(3), 968-980. https://doi. org/10.1016/j.neuroimage.2006.01.021

Doucet, G. E., Rasgon, N., McEwen, B. S., Micali, N., \& Frangou, S. (2017). Elevated body mass index is associated with increased integration and reduced cohesion of sensory-driven and internally guided resting-state functional brain networks. Cerebral Cortex, 1-10. https://doi.org/10.1093/cercor/bhx008

Etkin, A., Egner, T., Peraza, D. M., Kandel, E. R., \& Hirsch, J. (2006). Resolving emotional conflict: A role for the rostral anterior cingulate cortex in modulating activity in the Amygdala. Neuron, 51(6), 871882. https://doi.org/10.1016/j.neuron.2006.07.029

Filippini, N., MacIntosh, B. J., Hough, M. G., Goodwin, G. M., Frisoni, G. B., Smith, S. M., ... Mackay, C. E. (2009). Distinct patterns of brain activity in young carriers of the APOE-epsilon4 allele. Pnas, 106(17), 7209-7214. https://doi.org/10.1073/pnas.0811879106

Fischl, B., \& Dale, A. M. (2000). Measuring the thickness of the human cerebral cortex from magnetic resonance images. Proceedings of the National Academy of Sciences, 97(20), 11050-11055. https://doi. org/10.1073/pnas.200033797

Fischl, B., Liu, A., \& Dale, A. M. (2001). Automated manifold surgery: Constructing geometrically accurate and topologically correct models of the human cerebral cortex. IEEE Transactions on Medical Imaging, 20(1), 70-80. https://doi.org/10.1109/42.906426

Fischl, B., Van Der Kouwe, A., Destrieux, C., Halgren, E., Ségonne, F., Salat, D. H., ... Dale, A. M. (2004). Automatically parcellating the human cerebral cortex. Cerebral Cortex, 14(1), 11-22. https://doi. org/10.1093/cercor/bhg087

Friedman, N. P., Miyake, A., Corley, R. P., Young, S. E., DeFries, J. C., \& Hewitt, J. K. (2006). Not all executive functions are related to intelligence. Psychological Science, 17(2), 172-179. https://doi.org/10. $1111 / j .1467-9280.2006 .01681 . x$

Ghashghaei, H. T., Hilgetag, C. C., \& Barbas, H. (2007). Sequence of information processing for emotions based on the anatomic dialogue between prefrontal cortex and amygdala. NeuroImage, 34(3), 905923. https://doi.org/10.1016/j.neuroimage.2006.09.046

Gonzalez Andino, S. L., \& Grave de Peralta Menendez, R. (2012). Coding of saliency by ensemble bursting in the amygdala of primates. Frontiers in Behavioral Neuroscience, 6. https://doi.org/10. 3389/fnbeh.2012.00038

Gottfredson, L. S. (1998). The general intelligence factor. Scientific American Presents, 9(4), 24-29. Article

Goulden, N., Khusnulina, A., Davis, N. J., Bracewell, R. M., Bokde, A. L., McNulty, J. P., \& Mullins, P. G. (2014). The salience network is responsible for switching between the default mode network and the central executive network: Replication from DCM. NeuroImage, 99, 180-190. https://doi.org/10.1016/j.neuroimage.2014.05.052

Gunstad, J., Paul, R. H., Cohen, R. A., Tate, D. F., Spitznagel, M. B., \& Gordon, E. (2007). Elevated body mass index is associated with executive dysfunction in otherwise healthy adults. Comprehensive Psychiatry, 48(1), 57-61. https://doi.org/10.1016/j.comppsych. 2006.05.001

Gunstad, J., Paul, R. H., Cohen, R. A, Tate, D. F., Spitznagel, M. B., Grieve, S., \& Gordon, E. (2008). Relationship between body mass index and brain volume in healthy adults. The International Journal of Neuroscience, 118(11), 1582-1593. https://doi.org/10.1080/ 00207450701392282

Haber, S. N., \& Knutson, B. (2010). The reward circuit: Linking primate anatomy and human imaging. Neuropsychopharmacology, 35(1), 4-26. https://doi.org/10.1038/npp.2009.129

Haier, R. J., Jung, R. E., Yeo, R. A., Head, K., \& Alkire, M. T. (2004). Structural brain variation and general intelligence. NeuroImage, 23(1), 425-433. https://doi.org/10.1016/j.neuroimage.2004.04.025

Haier, R. J., Jung, R. E., Yeo, R. A., Head, K., \& Alkire, M. T. (2005). The neuroanatomy of general intelligence: Sex matters. NeuroImage, 25(1), 320-327. https://doi.org/10.1016/j. neuroimage.2004.11.019

Hamer, M., \& Chida, Y. (2009). Physical activity and risk of neurodegenerative disease: a systematic review of prospective evidence. Psychological Medicine, 39(1), 3-11. https://doi.org/10.1017/ S0033291708003681

Harrison, T. L., Shipstead, Z., Hicks, K. L., Hambrick, D. Z., Redick, T. S., \& Engle, R. W. (2013). Working memory training may increase working memory capacity but not fluid intelligence. Psychological Science, 24(12), 2409-2419. https://doi.org/10.1177/ 0956797613492984

Hassenstab, J. J., Sweet, L. H., Del Parigi, A., McCaffery, J. M., Haley, A. P., Demos, K. E., ... Wing, R. R. (2012). Cortical thickness of the cognitive control network in obesity and successful weight loss maintenance: a preliminary MRI study. Psychiatry Research, 202(1), 77-9. https://doi.org/10.1016/j.pscychresns.2011.09.008

Hayes, A. (2013). Introduction to mediation, moderation, and conditional process analysis. New York, NY: Guilford, 3-4. doi:978-1-60918230-4

Heitz, R. P., Redick, T. S., Hambrick, D. Z., Kane, M. J., Conway, A. R. A., \& Engle, R. W. (2006). Working memory, executive function, and general fluid intelligence are not the same. Behavioral and Brain Sciences, 29(2), 135-136. https://doi.org/10.1017/ S0140525X06319036

Hillman, C. H., Erickson, K. I., \& Kramer, A. F. (2008). Be smart, exercise your heart: exercise effects on brain and cognition. Nat Rev Neurosci., 9(1), 58-65. https://doi.org/10.1038/nrn2298

Hong, S., \& Hikosaka, O. (2008). The globus pallidus sends rewardrelated signals to the lateral habenula. Neuron, 60(4), 720-729. https://doi.org/10.1016/j.neuron.2008.09.035

Hung, C.-S., Yang, C.-Y., Hsieh, H.-J., Wei, J.-N., Ma, W.-Y., \& Li, H.-Y. (2012). BMI correlates better to visceral fat and insulin sensitivity than BAI. Obesity, 20(6), 1141-1141. https://doi.org/10.1038/oby. 2012.86

Hunot, C., Fildes, A., Croker, H., Llewellyn, C. H., Wardle, J., \& Beeken, R. J. (2016). Appetitive traits and relationships with BMI in adults: Development of the adult eating behaviour questionnaire. Appetite, 105, 356-363. https://doi.org/10.1016/j.appet.2016.05.024

Jaeggi, S. M., Buschkuehl, M., Jonides, J., \& Perrig, W. J. (2008). Improving fluid intelligence with training on working memory. Proceedings of the National Academy of Sciences of the United States of America, 105(19), 6829-6833. https://doi.org/10.1073/ pnas.0801268105

Jasinska, A. J., Yasuda, M., Burant, C. F., Gregor, N., Khatri, S., Sweet, M., \& Falk, E. B. (2012). Impulsivity and inhibitory control deficits 
are associated with unhealthy eating in young adults. Appetite, 59(3), 738-747. https://doi.org/10.1016/j.appet.2012.08.001

Jenkinson, M., Bannister, P., Brady, M., \& Smith, S. (2002). Improved optimization for the robust and accurate linear registration and motion correction of brain images. NeuroImage, 17(2), 825-841. https://doi.org/10.1016/S1053-8119(02)91132-8

Jilka, S. R., Scott, G., Ham, T., Pickering, A., Bonnelle, V., Braga, R. M., ... Sharp, D. J. (2014). Damage to the salience network and interactions with the default mode network. Journal of Neuroscience, 34(33), 10798-10807. https://doi.org/10.1523/JNEUROSCI.051814.2014

Jung, R. E., \& Haier, R. J. (2007). The Parieto-Frontal Integration Theory (P-FIT) of intelligence: converging neuroimaging evidence. Behavioral and Brain Sciences, 30(2), 135-54. https://doi.org/10. 1017/S0140525X07001185

Kanazawa, S. (2014). Intelligence and obesity: which way does the causal direction go?. Current Opinion in Endocrinology, Diabetes, and Obesity, 21(5), 339-344. https://doi.org/10.1097/MED. 0000000000000091

Karlsson, H. K., Tuulari, J. J., Hirvonen, J., Lepomäki, V., Parkkola, R., Hiltunen, J., ... Nummenmaa, L. (2013). Obesity is associated with white matter atrophy: A combined diffusion tensor imaging and voxel-based morphometric study. Obesity, 21(12), 2530-2537. https://doi.org/10.1002/oby.20386

Kennedy, J. T., Collins, P. F., \& Luciana, M. (2016). Higher adolescent body mass index is associated with lower regional gray and white matter volumes and lower levels of positive emotionality. Frontiers in Neuroscience. https://doi.org/10.3389/fnins.2016.00413

Kennerley, S. W., \& Walton, M. E. (2011). Decision making and reward in frontal cortex: Complementary evidence from neurophysiological and neuropsychological studies. Behavioral Neuroscience, 125(3), 297-317. https://doi.org/10.1037/a0023575

Keys, A., Fidanza, F., Karvonen, M. J., Kimura, N., \& Taylor, H. L. (1972). Indices of relative weight and obesity. Journal of Chronic Diseases, 25(6-7), 329-343. https://doi.org/10.1016/0021-9681(72) 90027-6

Kopelman, P. G. (2000). Obesity as a medical problem. Nature, 404(6778), 635-643. https://doi.org/10.1038/35007508

Kopelman, P. G. (2007). Health risks associated with overweight and obesity. Obesity Reviews : An Official Journal of the International Association for the Study of Obesity, 8 Suppl 1(11), 13-17. https:// doi.org/10.1111/j.1467-789X.2007.00311.x

Kullmann, S., Heni, M., Veit, R., Ketterer, C., Schick, F., Häring, H. U., ... Preissl, H. (2012). The obese brain: Association of body mass index and insulin sensitivity with resting state network functional connectivity. Human Brain Mapping, 33(5), 1052-1061. https://doi. org $/ 10.1002 / \mathrm{hbm} .21268$

Li, C.S., \& Sinha, R. (2008). Inhibitory control and emotional stress regulation: Neuroimaging evidence for frontal-limbic dysfunction in psycho-stimulant addiction. Neuroscience and Biobehavioral Reviews. https://doi.org/10.1016/j.neubiorev.2007.10.003

Lips, M. A., Wijngaarden, M. A., Van Der Grond, J., Van Buchem, M. A., De Groot, G. H., Rombouts, S. A. R. B., ... Veer, I. M. (2014). Resting-state functional connectivity of brain regions involved in cognitive control, motivation, and reward is enhanced in obese females. American Journal of Clinical Nutrition, 100(2), 524-531. https://doi.org/10.3945/ajen.113.080671

Marqués-Iturria, I., Pueyo, R., Garolera, M., Segura, B., Junqué, C., García-García, I., ... Jurado, M. Á. (2013). Frontal cortical thinning and subcortical volume reductions in early adulthood obesity. Psychiatry Research - Neuroimaging, 214(2), 109-115. https://doi. org/10.1016/j.pscychresns.2013.06.004

McAdams, M. A., Van Dam, R. M., \& Hu, F. B. (2007). Comparison of self-reported and measured BMI as correlates of disease markers in US adults. Obesity (Silver Spring, Md.), 15(1), 188-96. https://doi. org/10.1038/oby.2007.504
McDaniel, M. A. (2005). Big-brained people are smarter: A metaanalysis of the relationship between in vivo brain volume and intelligence. Intelligence, 33(4), 337-346. https://doi.org/10.1016/j. intell.2004.11.005

Medic, N., Ziauddeen, H., Ersche, K. D., Farooqi, I. S., Bullmore, E. T., Nathan, P. J., ... Fletcher, P. C. (2016). Increased body mass index is associated with specific regional alterations in brain structure. International Journal of Obesity (2005), 40(7), 1177-82. https:// doi.org/10.1038/ijo.2016.42

Menary, K., Collins, P. F., Porter, J. N., Muetzel, R., Olson, E. A., Kumar V., ... Luciana, M. (2013). Associations between cortical thickness and general intelligence in children, adolescents and young adults. Intelligence, 41(5), 597-606. https://doi.org/10.1016/j.intell.2013. 07.010

Mennes, M., Biswal, B. B., Castellanos, F. X., \& Milham, M. P. (2013). Making data sharing work: The FCP/INDI experience. NeuroImage, 82, 683-691. https://doi.org/10.1016/j.neuroimage.2012.10.064

Mokdad, A. H., Ford, E. S., Bowman, B. A., Dietz, W. H., Vinicor, F., Bales, V. S., \& Marks, J. S. (2003). Prevalence of obesity, diabetes, and obesity-related health risk factors, 2001. JAMA, 289(1), 76-79. https://doi.org/10.1001/jama.289.1.76

Morey, R. A., Petty, C. M., Xu, Y., Pannu Hayes, J., Wagner, H. R., Lewis, D. V., ... McCarthy, G. (2009). A comparison of automated segmentation and manual tracing for quantifying hippocampal and amygdala volumes. NeuroImage, 45(3), 855-866. https://doi.org/ 10.1016/j.neuroimage.2008.12.033

Munakata, Y., Herd, S. A., Chatham, C. H., Depue, B. E., Banich, M. T., \& O'Reilly, R. C. (2011). A unified framework for inhibitory control. Trends in Cognitive Sciences. https://doi.org/10.1016/j.tics. 2011.07.011

Neisser, U., Boodoo, G., Bouchard Jr., T. J., Boykin, A W., Brody, N., Ceci, S. J., ... Urbina, S. (1996). Intelligence: Knowns and unknowns. American Psychologist, 51(2), 77-101. https://doi.org/10. 1037/0003-066X.51.2.77

Nichols, T. E., \& Holmes, A. P. (2001). Nonparametric permutation tests for functional neuroimaging: A primer with examples. Human Brain Mapping, 15, 1-25. https://doi.org/10.1002/hbm.1058

Nooner, K. B., Colcombe, S. J., Tobe, R. H., Mennes, M., Benedict, M. M., Moreno, A. L., ... Milham, M. P. (2012). The NKI-Rockland sample: A model for accelerating the pace of discovery science in psychiatry. Frontiers in Neuroscience. https://doi.org/10.3389/fnins. 2012.00152

Ozier, A. D., Kendrick, O. W., Leeper, J. D., Knol, L. L., Perko, M., \& Burnham, J. (2008). Overweight and obesity are associated with emotion- and stress-related eating as measured by the eating and appraisal due to emotions and stress questionnaire. Journal of the American Dietetic Association, 108(1), 49-56. https://doi.org/10. 1016/j.jada.2007.10.011

Patenaude, B., Smith, S. M., Kennedy, D. N., \& Jenkinson, M. (2011). A Bayesian model of shape and appearance for subcortical brain segmentation. NeuroImage, 56(3), 907-922. https://doi.org/10.1016/j. neuroimage.2011.02.046

Petrides, M., \& Pandya, D. N. (2007). Efferent association pathways from the rostral prefrontal cortex in the macaque monkey. Journal of Neuroscience, 27(43), 11573-11586. https://doi.org/10.1523/ JNEUROSCI.2419-07.2007

Prentice, A M., \& Jebb, S. A. (2001). Beyond body mass index. Obesity Reviews : An Official Journal of the International Association for the Study of Obesity, 2(3), 141-147. https://doi.org/10.1046/j.1467789x.2001.00031.x

Pruim, R. H. R., Mennes, M., van Rooij, D., Llera, A., Buitelaar, J. K., \& Beckmann, C. F. (2015). ICA-AROMA: A robust ICA-based strategy for removing motion artifacts from fMRI data. NeuroImage, 112, 267-277. https://doi.org/10.1016/j.neuroimage.2015.02.064

Raji, C. A., Ho, A. J., Parikshak, N. N., Becker, J. T., Lopez, O. L., Kuller, L. H., ... Thompson, P. M. (2010). Brain structure and obesity. 
Human Brain Mapping, 31(3), 353-364. https://doi.org/10.1002/ hbm. 20870

Reuter, M., Rosas, H. D., \& Fischl, B. (2010). Highly accurate inverse consistent registration: A robust approach. NeuroImage, 53(4), 1181-1196. https://doi.org/10.1016/j.neuroimage.2010.07.020

Roth, G., \& Dicke, U. (2005). Evolution of the brain and intelligence. Trends in Cognitive Sciences. https://doi.org/10.1016/j.tics.2005.03. 005

Rozanski, A., Blumenthal, J. A., \& Kaplan, J. (1999). Impact of psychological factors on the pathogenesis of cardiovascular disease and implications for therapy. Circulation, 99, 2192-2217. https://doi. org/10.1161/01.cir.99.16.2192

Rueckert, D., Sonoda, L. I., Hayes, C., Hill, D. L. G., Leach, M. O., \& Hawkes, D. J. (1999). Nonrigid registration using free-form deformations: Application to breast MR images. IEEE Transactions on Medical Imaging, 18(8), 712-721. https://doi.org/10.1109/42. 796284

Ségonne, F., Dale, A. M., Busa, E., Glessner, M., Salat, D., Hahn, H. K., \& Fischl, B. (2004). A hybrid approach to the skull stripping problem in MRI. NeuroImage, 22(3), 1060-1075. https://doi.org/10. 1016/j.neuroimage.2004.03.032

Ségonne, F., Pacheco, J., \& Fischl, B. (2007). Geometrically accurate topology-correction of cortical surfaces using nonseparating loops. IEEE Transactions on Medical Imaging, 26(4), 518-529. https:// doi.org/10.1109/TMI.2006.887364

Sirin, S. R. S. (2005). Socioeconomic status and academic achievement : A meta-analytic review of research. American Educational Research Association, 75(3), 417-453. Retrieved from http://rer. sagepub.com/content $/ 75 / 3 / 417$.short

Sled, J. G., Zijdenbos, A P., \& Evans, A C. (1998). A nonparametric method for automatic correction of intensity nonuniformity in MRI data. IEEE Transactions on Medical Imaging, 17(1), 87-97. https://doi.org/10.1109/42.668698

Smith, S. M. (2002). Fast robust automated brain extraction. Human Brain Mapping, 17(3), 143-155. https://doi.org/10.1002/hbm. 10062

Smith, S. M., Jenkinson, M., Johansen-Berg, H., Rueckert, D., Nichols, T. E., Mackay, C. E., ... Behrens, T. E. J. (2006). Tract-based spatial statistics: Voxelwise analysis of multi-subject diffusion data. NeuroImage, 31(4), 1487-1505. https://doi.org/10.1016/j. neuroimage.2006.02.024

Smith, S. M., Jenkinson, M., Woolrich, M. W., Beckmann, C. F., Behrens, T. E. J., Johansen-Berg, H., ... Matthews, P. M. (2004). Advances in functional and structural MR image analysis and implementation as FSL. Neuroimage, 23(SUPPL. 1), S208-S219. https://doi.org/10.1016/j.neuroimage.2004.07.051

Smith, S. M., \& Nichols, T. E. (2009). Threshold-free cluster enhancement: Addressing problems of smoothing, threshold dependence and localisation in cluster inference. NeuroImage, 44(1), 83-98. https://doi.org/10.1016/j.neuroimage.2008.03.061

Song, M., Zhou, Y., Li, J., Liu, Y., Tian, L., Yu, C., \& Jiang, T. (2008). Brain spontaneous functional connectivity and intelligence. NeuroImage, 41(3), 1168-1176. https://doi.org/10.1016/j. neuroimage.2008.02.036

Spearman, C. (1904). \&quot; General Intelligence,\&quot; Objectively Determined and Measured. The American Journal of Psychology, 15(2), 201-292. https://doi.org/10.2307/1412107

Spielberg, J. M., Miller, G. A., Heller, W., \& Banich, M. T. (2015). Flexible brain network reconfiguration supporting inhibitory control. Proceedings of the National Academy of Sciences, 112(32), 10020-10025. https://doi.org/10.1073/pnas.1500048112

Sridharan, D., Levitin, D. J., \& Menon, V. (2008). A critical role for the right fronto-insular cortex in switching between central-executive and default-mode networks. Proceedings of the National Academy of Sciences, 105(34), 12569-12574. https://doi.org/10.1073/pnas. 0800005105
Stanek, K. M., Grieve, S. M., Brickman, A. M., Korgaonkar, M. S., Paul, R. H., Cohen, R. A, \& Gunstad, J. J. (2011). Obesity is associated with reduced white matter integrity in otherwise healthy adults. Obesity (Silver Spring, Md.), 19(3), 500-504. https://doi.org/10. 1038/oby.2010.312

Stroop, J. R. (1935). Stroop color word test. Journal Experiment of Physiology, (18), 643-662. https://doi.org/10.1007/978-0-38779948-3

Suchanek, P., Kralova Lesna, I., Mengerova, O., Mrazkova, J., Lanska, V., \& Stavek, P. (2012). Which index best correlates with body fat mass: BAI, BMI, waist or WHR? Neuroendocrinology Letters (Vol. 33 , pp. 78-82).

Taki, Y., Kinomura, S., Sato, K., Inoue, K., Goto, R., Okada, K., ... Fukuda, H. (2008). Relationship between body mass index and gray matter volume in 1,428 healthy individuals. Obesity, 16(16), 119 124. https://doi.org/10.1038/oby.2007.4

Trost, S. G., Owen, N., Bauman, A. E., Sallis, J. F., \& Brown, W. (2002). Correlates of adults' participation in physical activity: Review and update. Medicine and Science in Sports and Exercise, 34(12), 19962001. https://doi.org/10.1097/00005768-200212000-00020

Ursache, A., \& Noble, K. G. (2016). Socioeconomic status, white matter, and executive function in children. Brain and Behavior, 6(10), 1-13. https://doi.org/10.1002/brb3.531

Vakhtin, A. A., Ryman, S. G., Flores, R. A., \& Jung, R. E. (2014). Functional brain networks contributing to the Parieto-Frontal Integration Theory of Intelligence. NeuroImage, 103, 349-354. https://doi.org/10.1016/j.neuroimage.2014.09.055

Veit, R., Kullmann, S., Heni, M., Machann, J., Häring, H. U., Fritsche, A., \& Preissl, H. (2014). Reduced cortical thickness associated with visceral fat and BMI. NeuroImage: Clinical, 6, 307-311. https:// doi.org/10.1016/j.nicl.2014.09.013

Verstynen, T. D., Weinstein, A. M., Schneider, W. W., Jakicic, J. M., Rofey, D. L., \& Erickson, K. I. (2012). Increased body mass index is associated with a global and distributed decrease in white matter microstructural integrity. Psychosomatic Medicine, 74(7), 682-690. https://doi.org/10.1097/PSY.0b013e318261909c

Volkow, N. D., Wang, G.-J., Telang, F., Fowler, J. S., Goldstein, R. Z., Alia-Klein, N., ... Pradhan, K. (2009). Inverse association between BMI and prefrontal metabolic activity in healthy adults. Obesity, 17(1), 60-65. https://doi.org/10.1038/oby.2008.469

Walther, K., Birdsill, A. C., Glisky, E. L., \& Ryan, L. (2010). Structural brain differences and cognitive functioning related to body mass index in older females. Human Brain Mapping, 31(7), 1052-1064. https://doi.org/10.1002/hbm.20916

Wang, F., Kalmar, J. H., He, Y., Jackowski, M., Chepenik, L. G., Edmiston, E. E., ... Blumberg, H. P. (2009). Functional and structural connectivity between the perigenual anterior cingulate and amygdala in bipolar disorder. Biological Psychiatry, 66(5), 516521. https://doi.org/10.1016/j.biopsych.2009.03.023

Wang, Y., \& Beydoun, M. A. (2007). The obesity epidemic in the United States - Gender, age, socioeconomic, racial/ethnic, and geographic characteristics: A systematic review and meta-regression analysis. Epidemiologic Reviews, 29(1), 6-28. https://doi.org/10.1093/epirev/ $\mathrm{mxm} 007$

Ward, M. A, Carlsson, C. M., Trivedi, M. A, Sager, M. A, \& Johnson, S. C. (2005). The effect of body mass index on global brain volume in middle-aged adults: a cross sectional study. BMC Neurology, 5, 23. https://doi.org/10.1186/1471-2377-5-23

Wechsler, D. (2011). Wechsler Abbreviated Scale of Intelligence-Second Edition. Wechsler Abbreviated Scale of Intelligence-Second Edition (WASI-II).

WHO. (2000). Obesity: preventing and managing the global epidemic. Report of a WHO consultation. World Health Organization Technical Report Series, 894, 1-253. https://doi.org/10.1016/ S0140-6736(57)91352-1 
Wickett, J. C., Vernon, P. A, \& Lee, D. H. (2000). Relationships between factors of intelligence and brain volume. Personality and Individual Differences, 29(6), 1095-1122. https://doi.org/10.1016/S01918869(99)00258-5

Willeumier, K. C., Taylor, D. V., \& Amen, D. G. (2011). Elevated BMI is associated with decreased blood flow in the prefrontal cortex using SPECT imaging in healthy adults. Obesity, 19, 1095-1097. https:// doi.org/10.1038/oby.2011.16

Winkler, A. M., Ridgway, G. R., Webster, M. A., Smith, S. M., \& Nichols, T. E. (2014). Permutation inference for the general linear model. NeuroImage, 92, 381-397. https://doi.org/10.1016/j. neuroimage.2014.01.060

Witelson, S. F., Beresh, H., \& Kigar, D. L. (2006). Intelligence and brain size in 100 postmortem brains: Sex, lateralization and age factors. Brain, 129(2), 386-398. https://doi.org/10.1093/brain/awh696

Woolrich, M. W., Jbabdi, S., Patenaude, B., Chappell, M., Makni, S., Behrens, T., ... Smith, S. M. (2009). Bayesian analysis of neuroimaging data in FSL. NeuroImage, 45(1 Suppl). https://doi.org/10. 1016/j.neuroimage.2008.10.055

Xu, J., Li, Y., Lin, H., Sinha, R., \& Potenza, M. N. (2013). Body mass index correlates negatively with white matter integrity in the fornix and corpus callosum: A diffusion tensor imaging study. Human
Brain Mapping, 34(5), 1044-1052. https://doi.org/10.1002/hbm. 21491

Yeo, B. T., Krienen, F. M., Sepulcre, J., Sabuncu, M. R., Lashkari, D., Hollinshead, M., ... Buckner, R. L. (2011). The organization of the human cerebral cortex estimated by intrinsic functional connectivity. Journal of Neurophysiology, 106(3), 1125-1165. https://doi.org/10. 1152/jn.00338.2011

Yi, S.-W., Ohrr, H., Shin, S.-A., \& Yi, J.-J. (2015). Sex-age-specific association of body mass index with all-cause mortality among 12.8 million Korean adults: a prospective cohort study. International Journal of Epidemiology, (July), 1-10. https://doi. org/10.1093/ije/dyv138

Yokum, S., Ng, J., \& Stice, E. (2012). Relation of regional gray and white matter volumes to current BMI and future increases in BMI: a prospective MRI study. International Journal of Obesity (2005), 36(5), 656-64. https://doi.org/10.1038/ijo.2011.175

Zelazo, P. D., \& Cunningham, W. A. (2007). Executive function: mechanisms underlying emotion regulation. In Handbook of emotion regulation (pp. 135-158). Retrieved from http://psycnet.apa.org/ psycinfo/2007-01392-007\%5Cnpapers3://publication/uuid/ 28AE6820-F540-4881-AA55-A72276388A32 